\title{
Sports Industry and Economic Development Correlation Degree Research Based on Grey Relational Degree Model
}

\author{
Bing Zhang ${ }^{1}$, Kunling Qin ${ }^{2}$, Xuefeng Cao ${ }^{1}$ \\ ${ }^{1}$ Institute of Physical Education, Huanggang Normal University, Huangzhou 438000, Hubei, China \\ ${ }^{2}$ Yidu Gaobazhou Middle School, Yidu 443300, Hubei, China \\ tiyuxi@qq.com
}

\begin{abstract}
Sports industry going through nearly half a century development since 1950s until now, which has already begun to take shape in worldwide sports industry. Especially for lots of developed countries in Europe and America, sports industry has already become occupied big advantage that belongs to economic system core industry in national economy. By comparing, Chinese sports industry started later, though sports industry proportion of economy is constantly increasing, it still falls behind developed countries. The paper makes research on sports industry and economic development relations by reasonable defining sports industry and national economy indicators. Adopt mathematical method looking for sports industry and economy correlation effects, and makes research on sports industry structure optimization development and economic way propelling to sports industry development. Do initialize handling with data, solve correlation degree through establishing sports industry and economy grey mathematical model. It gets conclusion that sports industry can better propel to economic development, especially it has highest correlation degree with GDP, and economic development can reversely propel to economic development and form into virtuous circle.
\end{abstract}

Keywords: sports industry, economic development, structural optimization

\section{INTRODUCTION}

Under new period, Chinese economy should change growth pattern, which needs to make adjustment on industrial structure and propel to economic healthy development, sports industry as sunrise industry, it has important significance in propelling to economic development. No doubt, sports development surely propels to economic development, and economic growth surely will also have huge effects on sports industry development. But how are their promoting degrees like? Correctly solve the problem has important practical significances in planning scientific reasonable sports industrial policies, designing reasonable layers sports industry structure and rapidly propelling to sports industry development (BING Zhang, 2014).

In sports industry and economic correlations, scholars have made a great deal researches. Among them, Yang Qian researched sports industry and economic relations by grey relative analysis method, she pointed out that sports industry sub-factor fitness entertainment had highest correlation degree with economy, and national economy played strong leading role in sports industry development (LUO Hua-min, 2005). Tong Ying-Juan applied growth factors analysis method to deeply research on sports industry development external factors and economic factors; she got layout pattern and layout economic means based on eastern region sports industry. Hu Cheng-Hong etc. based on grey mathematical theory, established evaluation model for sports industry structure, and carried out sports industry structural research with Sichuan province as an example; they thought it should lead relative industrial development on the basis of sports industry (BING Zhang, Duo Tang, 2013). Yu Shou-Wen investigated developed countries sports industry structural form and economic structural effects by collecting information, he thought that sports industry was economic growth newly-developed part, it could drive sports industry chain and economic rapidly development by mutual correlations. Zhu Jie applied factor analysis, on the basis of Chinese eastern each province practical situations, she did research on sports industry development influence factors and put forward sports industry development promoting methods and suggestions (MA Yufeng, 2001).

The paper on the basis of previous researches, by referencing, it applies grey correlation mathematical model to make further research on sports industry and economy relations. Calculate correlation degree 
as well as correlation matrix analysis of sports industry to economic development importance. By advantage analysis, it finds out promoting sports industry best economic factors, and gets sports industry output value to GDP correlation degree, consumption index per capita to sports industry development correlation effects and other relations.

\section{Sports Industry and Economic Grey Mathematical Model Correlation DEGREE}

Relations between sports industry and economy are intricate, mutually effected, their each kind of economic indicator and sports industry inner relations, structure, as well as features conform to grey mathematical model. Due to sports industry and economy detailed parameters correlation form is not exactly, it belongs to grey system. Grey system is the term of system that information is not completely, partial known and partial unknown in system. This paper starts from grey system original feature grey, researches on information greatly lacking of clear correlations system. Grey system can better fit and find out things grey relations, establish sports industry sub-factor and economic subfactor correlations, and hereby solve and handle with sports industry and economy correlations. Calculate sports industry sub factors and economy sub factors correlation degree, sort out and solve correlation degree matrix to process with advantage analysis (HUANG Sheng-fang et al., 2000).

\subsection{Indicators Selection}

There are many sports industry development status indicator factors, the paper selects most representative three factors that are respectively sports industry output, sports consumption value and number of sports industry staff. The three factors basically can represent Chinese sports industry development form levels; they are defined as sports industry three sub factors.

Economic development sub factors selection. (1)Gross domestic product is the effect of sports industry development, it is often used for measuring a country or regional economic development level, is an important economic indicator.(2)Tertiary industry output, in tertiary industry, sports industry is one important part, it plays an important role in tertiary industry, sports industry development will surely also have tertiary industry development.(3) Per capita GDP.(4) Per capita GDP high, the two indicators mark people consumption potential and consumption ability, which reflects the public consumption ability in sports aspect.(5) Per capita consumption expenditure.(6) Per capita disposable income such two indicators have larger relations with sports industry consumption, which decide national consumption potential purchasing potential that have important effects on sports industry consumption. All have larger correlations. Number of Tertiary industry employees, it plays assignable roles in promoting economic development, sports industry as an important branch of three main industries, employees in tertiary industry reflects industry structure sizes, meanwhile it reflects sports industry development level.

Table1: Factor list

\begin{tabular}{|l|l|}
\hline Indicator & Sub-factor \\
\hline \multirow{4}{*}{ Sports industry } & Sports industry output \\
\cline { 2 - 2 } & Sports consumption value \\
\cline { 2 - 2 } & Number of sports industry staff \\
\hline \multirow{5}{*}{ Economic development } & Gross domestic product \\
\cline { 2 - 2 } & Tertiary industry output \\
\cline { 2 - 2 } & Per capita GDP \\
\cline { 2 - 2 } & Per capita consumption expenditure \\
\cline { 2 - 2 } & Per capita disposable income \\
\cline { 2 - 2 } & Number of Tertiary industry employees \\
\hline
\end{tabular}

\subsection{Correlation Analysis and Solution}

Correlation degree analysis method is put forward by grey system theory. It can make calculation and handling with incomplete information or imperfect information, which is suitable to the paper researched sports industry and economic development correlations. Correlation degree geometric significance is similarity degree after factor converted into function images. Its calculated amounts are less and not prone to appear correlation degree quantization result and qualitative analysis inconsistent status. As a developing and changing system, correlation analysis actually is dynamical process development trend quantization comparative analysis. 
Bing Zhang et al.

Sports industry and economic development factors data from 1997 to 2007 is as following Table 2, from which $x_{1}$ represents sports output, $x_{2}$ represents sports consumption value, $x_{3}$ represents number of sports industry staff。 $y_{1}$ represents gross domestic product GDP, $y_{2}$ represents tertiary industry output, $y_{3}$ represents domestic per capita GDP, $y_{4}$ represents urban per capita consumption expenditure, $y_{5}$ represents urban per capita disposable income, $y_{6}$ represents number of tertiary industry employees. Make correlation analysis of above each factor sequence, it needs to define reference sequence and comparison sequence, now regulates sports industry sub-factor as comparison sequence, economic development sub-factor as reference sequence, solve comparison sequence and reference sequence correlation degree.

Table 2: Each factor years of data

\begin{tabular}{|c|c|c|c|c|c|c|c|}
\hline \multicolumn{2}{|c|}{ Year } & 1997 & 1998 & 1999 & 2000 & 2001 & 2002 \\
\hline Sports output & $x_{1}$ & 157.95 & 168.8 & 179.35 & 198.43 & 219.31 & 240.67 \\
\hline Sports consumption & $x_{2}$ & 2562.12 & 2687.96 & 2818.2 & 3014 & 3129.72 & 3250.28 \\
\hline Number of sports industry staff & $x_{3}$ & 471 & 478 & 482 & 488.09 & 493.04 & 193.25 \\
\hline GDP & $y_{1}$ & 78973.03 & 84402.28 & 89677.05 & 99214.55 & 109655.2 & 120332.7 \\
\hline Tertiary industry output & $y_{2}$ & 26988.15 & 30580.47 & 33873.44 & 38713.95 & 44361.61 & 49898.9 \\
\hline Per capita GDP & $y_{3}$ & 6420.18 & 6796.03 & 7158.5 & 7857.68 & 8621.71 & 9398.05 \\
\hline Per capita consumption & $y_{4}$ & 5832 & 6109 & 6405 & 6850 & 7113 & 7387 \\
\hline Per capita disposable income & $y_{5}$ & 5160.3 & 5425.1 & 5854.02 & 6280 & 6859.6 & 7702.8 \\
\hline Number of Tertiary industry employees & $y_{6}$ & 18432 & 18860 & 19205 & 19823 & 20228 & 21090 \\
\hline Year & & 2003 & 2004 & 2005 & 2006 & 2007 & \\
\hline Sports output & $x_{1}$ & 271.65 & 319.76 & 366.43 & 423.847 & 199.06 & \\
\hline Sports consumption & $x_{2}$ & 3476.44 & 3818.76 & 4140.4 & 4586.12 & 5216.2 & \\
\hline Number of sports industry staff & $x_{3}$ & 493.15 & 493.2 & 493.17 & 493.18 & 493.18 & \\
\hline GDP & $y_{1}$ & 135822.8 & 159878.3 & 183217.4 & 211923.5 & 249529.9 & \\
\hline Tertiary industry output & $y_{2}$ & 56004.73 & 64561.29 & 73432.87 & 84721.4 & 100053.5 & \\
\hline Per capita GDP & $y_{3}$ & 10541.97 & 12335.58 & 14053 & 16165 & 18934 & \\
\hline Per capita consumption & $y_{4}$ & 7901 & 8679 & 9410 & 10423 & 11855 & \\
\hline Per capita disposable income & $y_{5}$ & 8472.2 & 9421.6 & 10493 & 11759.5 & 13785.8 & \\
\hline Number of Tertiary industry employees & $y_{6}$ & 21809 & 23011 & 23771 & 24614 & 24917 & \\
\hline
\end{tabular}

Adopt grey mathematical model solving correlation degree, convert sports industry and economic development correlation into mathematical problems, in Table 2 sports industry data expressed by matrix A, it gets:

$$
\mathrm{A}=\left(\begin{array}{cccccc}
157.95 & 168.8 & 179.35 & \ldots & \ldots & 199.06 \\
2562.12 & 2687.96 & 28118.2 & \ldots & \ldots & 5216.2 \\
471 & 478 & 482 & \ldots & \ldots & 493.18
\end{array}\right)
$$

Define $\mathrm{A}$ as original data comparison matrix. In matrix $\mathrm{A}$, row represents sequence $x_{1}, x_{2}, x_{3}$, column represents year.

In Table 2 economic development factor is expressed by matrix B as: 


$$
\mathrm{B}=\left(\begin{array}{ccccc}
78973.03 & 84402.28 & 89677.05 & \ldots & 249529.9 \\
26988.15 & 30580.47 & 33873.44 & \ldots & 100053.5 \\
6420.18 & 6796.03 & 7158.5 & \ldots & 18934 \\
5832 & 6109 & 6405 & \ldots & 11855 \\
5160.3 & 5425.1 & 5854.02 & \ldots & 13785.8 \\
18432 & 18860 & 19205 & \ldots & 24917
\end{array}\right)
$$

Define B as original data comparison matrix. In matrix B, row represents sequence $y_{1}, y_{2}, y_{3}$, $y_{4}, y_{5}, y_{6}$ column represents year.

1. At first carry out data transformation

Because collected original data with different dimensions that have no comparability, to ensure modeling result accuracy, it should proceed with data transformation. Method is as following:

Define 1 Ordered sequence

$$
x=(x(1), x(2), \cdots x(n))
$$

And then call map

$$
\begin{gathered}
f: x \rightarrow y \\
f(x(k))=y(k), k=1,2, \cdots n
\end{gathered}
$$

It is sequence $x$ to sequence $y$ data transformation. Its data transformation has:initialization transformation, mean transformation, percentage transformation, multiple transformation, normalization transformation, maximum range transformation, interval values transformation and so on. Here it adopts initialization transformation:

$$
f(x(k))=\frac{x(k)}{x(1)}=y(k), k=1,2, \cdots n \operatorname{nand} x(1) \neq 0
$$

That is $f$ initialization transformation. Make initialization transformation on matrix $\mathrm{A}$, adopt matrix form transformation.

Define transformation matrix $\mathrm{C}$ : Let matrix that converts original data matrix $\mathrm{A}$ into initial value matrix $\mathrm{D}$ is called transformation matrix. Expression is: $\mathrm{C} \bullet \mathrm{A}=\mathrm{D}$

Matrix C general expression is: $\mathrm{C}=\left(\begin{array}{cccc}1 / a_{11} & 0 & \cdots & 0 \\ 0 & 1 / a_{21} & \cdots & 0 \\ \vdots & \vdots & \ddots & \vdots \\ 0 & 0 & \cdots & 1 / a_{n 1}\end{array}\right)$

So make initialization transformation on $\mathrm{A}, \mathrm{B}$ by transformation matrix $\mathrm{C}$, it can get matrix:

$$
\begin{aligned}
\mathrm{D}=\mathrm{C} \bullet \mathrm{A} & =\left(\begin{array}{cccccc}
1.068 & 1.135 & \ldots & & \ldots & 1.260 \\
1.049 & 1.100 & \ldots & & \ldots & 2.036 \\
1.015 & 1.023 & \ldots & & \ldots & 1.047
\end{array}\right) \\
\mathrm{E} & =\mathrm{C} \bullet \mathrm{B}=\left(\begin{array}{ccccc}
1.069 & 1.136 & \ldots & 3.160 \\
1.133 & 1.255 & \ldots & 3.707 \\
1.056 & 1.115 & \ldots & 2.949 \\
1.047 & 1.098 & \ldots & 2.033 \\
1.051 & 1.134 & \ldots & 2.672 \\
1.023 & 1.042 & \ldots & 1.352
\end{array}\right)
\end{aligned}
$$

Matrix D is comparison matrix after grey theory data initialization, $\mathrm{E}$ is reference matrix after grey theory data initialization, every row is D one reference sequence. Column represents values from 
1998 to 2007.

Draw Figure 1 with data after initialization; observe sports industry sub factors and economic development six sub factors geometric figure, initially judge their correlations, and parameters to performance correlation degree sizes.

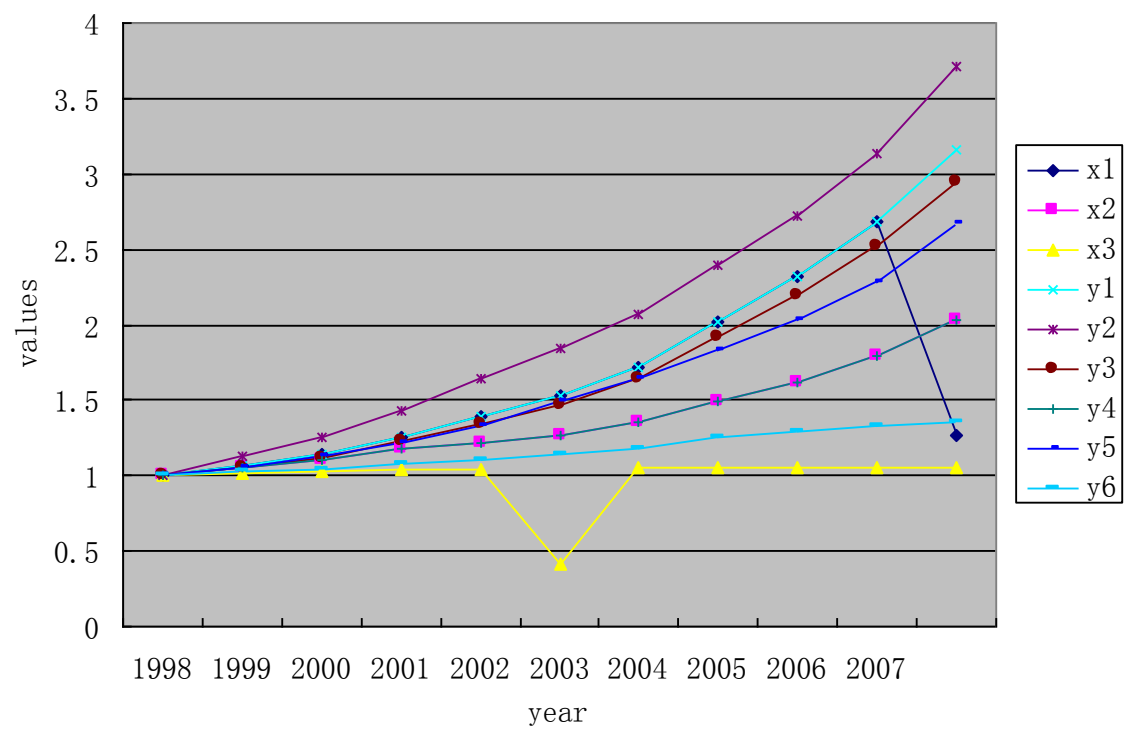

Figure1. Factor trend correlation figure

By above Figure 1 three qualitative analysis curve figure, it is clear that economic sub factors and sports industry sub factors trend approximate to consistency, there is similarity in figure, but each similar degree is different, which can roughly judge factors interaction sizes, but cannot make quantitative judgment on mutual correlation degrees sizes.

\section{Correlation coefficient solution}

Select reference sequence. Reference sequence in the paper is economic development sub factors. Other sequences are comparison sequence. Reference sequence:

$$
x_{0}=\left\{x_{0}(k) \mid k=1,2, \cdots n\right\}=\left(x_{0}(1), x_{0}(2) \cdots x_{0}(n)\right)
$$

Among them, $k$ is number of economic development sub factors, assume it has $m$ pieces of comparison sequence:

$$
x_{i}=\left\{x_{i}(k) \mid k=1,2, \cdots n\right\}=\left(x_{i}(1), x_{i}(2) \cdots x_{i}(n)\right), i=1,2, \cdots m
$$

Then it calls: $\xi_{i}(k)=\frac{\min _{s} \min _{t}\left|x_{0}(t)-x_{s}(t)\right|+\rho \max _{s} \max _{t}\left|x_{0}(t)-x_{s}(t)\right|}{\left|x_{0}(k)-x_{i}(k)\right|+\rho \max _{s} \max _{t}\left|x_{0}(t)-x_{s}(t)\right|}$

It is comparison sequence $x_{i}$ to reference sequence economic development sub factors ${ }^{x_{0}}$ at t moment correlation coefficient, from which $\rho \in[0,1]$ is resolution coefficient. In above formula, $\min \min \left|x_{0}(t)-x_{s}(t)\right| \cdot \max _{s} \max _{t}\left|x_{0}(t)-x_{s}(t)\right|$ are respectively two-level minimum difference and twolevel maximum difference.

Generally speaking, the bigger resolution ratio is, then the bigger resolution coefficient $\rho$ would be; the smaller resolution ratio is, and then the smaller $\rho$ would be, here the calculation takes $\rho=0.5$.

Correlation degree solution, correlation coefficient is a kind of indicator describing reference sequence and comparison sequence correlation degree at some time, due to each point has a correlation coefficient, it is not convenient to compare, so give correlation degree definition:

$$
r_{i}=\frac{1}{n} \sum_{k=1}^{n} \xi_{i}(k)
$$


It is sequence $x_{i}$ to reference sequence ${ }^{x_{0}}$ correlation degree. Correlation degree is concentrating each time correlation coefficient into an average value, which is also do collective handling with excess scattering information. Utilize correlation degree the concept, it can analyze and research sports industry and economic development correlation.

The solution, input initialized Table 2 data into formula(1), (2), it can get each sequence correlation degree by calculating.

Calculated MATLAB program is as following:

clc, clear

load x.txt

for $\mathrm{i}=1: 6$

$$
x(i,:)=x(i,:) / x(i, 1) \text {; }
$$

end

for $\mathrm{i}=6: 7$

$$
\mathrm{x}(\mathrm{i},:)=\mathrm{x}(\mathrm{i}, 1) \cdot \mathrm{x}(\mathrm{i},:) \text {; }
$$

end

data $=\mathrm{x}$;

$\mathrm{n}=\operatorname{size}($ data, 1$)$;

$\mathrm{ck}=\operatorname{data}(1,:) ; \mathrm{ml}=\operatorname{size}(\mathrm{ck}, 1)$;

bj=data $(2: n,:) ; m 2=\operatorname{size}(b j, 1)$;

for $\mathrm{i}=1: \mathrm{m} 1$

$$
\begin{aligned}
& \text { for } \mathrm{j}=1: \mathrm{m} 2 \\
& \mathrm{t}(\mathrm{j},:)=\operatorname{bj}(\mathrm{j},:)-\operatorname{ck}(\mathrm{i},:) ;
\end{aligned}
$$

end

$$
\begin{aligned}
& \mathrm{jc} 1=\min \left(\min \left(\operatorname{abs}\left(\mathrm{t}^{\prime}\right)\right)\right) ; \mathrm{jc} 2=\max \left(\max \left(\operatorname{abs}\left(\mathrm{t}^{\prime}\right)\right)\right) ; \\
& \mathrm{rho}=0.5 ; \\
& \mathrm{ksi}=(\mathrm{jc} 1+\mathrm{rho} * \mathrm{jc} 2) . /(\operatorname{abs}(\mathrm{t})+\mathrm{rho} * \mathrm{jc} 2) ; \\
& \mathrm{rt}=\operatorname{sum}\left(\mathrm{ksi} i^{\prime}\right) / \operatorname{size}(\mathrm{ksi}, 2) ; \\
& \mathrm{r}(\mathrm{i},:)=\mathrm{rt} ;
\end{aligned}
$$

end

$\mathrm{r}$

$[\mathrm{rs}$, rind $]=\operatorname{sort}(\mathrm{r}$, 'descend')

3. Correlation degree result

Respectively calculate sports industry sub factors and six reference sequence correlation degree, by calculation, it can get correlation degree matrix $r$, from which $r_{i j}$ represents sports industry factor $j$ to reference sequence economic development factor $i$ correlation degree, and express it with table, the result is as following Table 3:

Table 3: Correlation degree value

\begin{tabular}{|l|r|r|rr|}
\hline & $x_{1}$ & $x_{2}$ & & $x_{3}$ \\
\hline$y_{1}$ & 0.935709 & 0.75826 & 0.623493 \\
\hline$y_{2}$ & 0.821289 & 0.719224 & 0.62237 \\
\hline
\end{tabular}


Bing Zhang et al.

\begin{tabular}{|l|r|r|r|}
\hline \hline$y_{3}$ & 0.883461 & 0.787901 & 0.631907 \\
\hline$y_{4}$ & 0.637778 & 0.998874 & 0.605395 \\
\hline$y_{5}$ & 0.857613 & 0.823304 & 0.659658 \\
\hline$y_{6}$ & 0.665389 & 0.783647 & 0.814087 \\
\hline
\end{tabular}

\section{CONClusion}

Advantage analysis, by above Table 3, it is clear that the column of number of sports industry staff correlation degree value show minimum value, which shows that number of sports industry staff has no big effects on promoting economy. Sports output column correlation degree overall is the highest, which proves sports output can better promote economic development; especially it has highest correlation degree with GDP that is sports industry important effects on economic development. By advantage analysis, it finds out best economic factors that promote sports industry, and gets sports industry output has highest correlation degree with GDP. Per capita consumption index has strongest correlation effects on sports industry development, which means improving per capita consumption index can obviously improve sports industry sports consumption that plays positive roles in promoting sports industry development. Economic development rapidly growth can speed up sports industry development, sports industry development meanwhile can also promote economy further development, the two mutually promotion constructs virtuous circle industry system.

\section{REFERENCES}

[1] BING Zhang, (2014). 110m hurdles phased performance significance research based on SPSS regression analysis and GRA model. Journal of Chemical and Pharmaceutical Research, 6(2), 649-659.

[2] LUO Hua-min, (2005). Consideration on Perfecting Statistical Index System of Sports Industry in China[J].Journal of Shenyang Sport University, 24(4), 28-30, 33.

[3] BING Zhang, Duo Tang, (2013). Application of gray correlation theory for the impact factor analysis of the integrative development of the sports industry and tourism industry. International Journal of Applied Mathematics and Statistics, 51(21), 148-155.

[4] MA Yufeng, (2001). Research on the development of sports industry in Henan province[J]. Journal of Wuhan Institute of Physical Education, 35(1), 39-43.

[5] HUANG Sheng-fang et al., (2000). Initial study on statistical indices system of sports industry of China[J].Zhejiang Sport Science, 22(1), 1-5, 23. 